\title{
PICTORIAL REVIEW
}

\section{Diagnosis of colonic volvulus: findings on multidetector CT with three-dimensional reconstructions}

\author{
${ }^{1} \mathrm{C}$ VANDENDRIES, ${ }^{1} \mathrm{M}$ C JULLÈS, MD, ${ }^{1} \mathrm{I}$ BOULAY-COLETTA, MD, ${ }^{2} \mathrm{~J}$ LORIAU, MD and ${ }^{1} \mathrm{M}$ ZINS, MD \\ ${ }^{1}$ Department of Radiology, Groupe Hospitalier Paris Saint-Joseph, 185 Rue Raymond Losserand, 75014 Paris, France and \\ ${ }^{2}$ Department of Digestive Surgery, Groupe Hospitalier Paris Saint-Joseph, 185 Rue Raymond Losserand, 75014 Paris, \\ France
}

\begin{abstract}
Large bowel volvulus is a rare condition that can occur in patients who present with acute abdominal pain. Radiologists should be able to recognise its appearance on multidetector CT (MDCT) images so that the correct diagnosis can be made and catastrophic consequences can be avoided. In this article, we discuss and illustrate the MDCT and three-dimensional appearance of the various forms of large bowel volvulus. As MDCT allows the precise diagnosis of topography, mechanism and severity, this technique can provide an accurate assessment of large bowel volvulus.
\end{abstract}

\author{
Received 23 December \\ 2009 \\ Revised 2 March 2010 \\ Accepted 9 March 2010
}

DOI: $10.1259 / \mathrm{bjr} / 35714052$

(c) 2010 The British Institute of Radiology
A large bowel volvulus (LBV) is a twist of the bowel along its own mesentery, often resulting in a closed-loop obstruction. LBV accounts for 5\% of all organic largebowel obstructions and are most common among patients aged between 50 and 60 in North America and Western Europe [1, 2]. The reported incidence of the various forms of LBV in the urban Australian population is $59 \%$ for sigmoid volvulus (SV), $39 \%$ for cecal volvulus $(\mathrm{CV})$ and $2 \%$ for transverse colon volvulus [2]. Other rare forms account for less than $1 \%$ of cases. In our review of the radiology archives at our institution from August 2004 to August 2008, we encountered 23 LBVs: 13 cases of SV $(57 \%)$, six cases of CV $(26 \%)$, and four cases of other rare forms of the condition (17\%).

The diagnosis of LBV can be challenging because its clinical presentation has low specificity compared with other non-traumatic abdominal pain. Abdominal radiographs have been shown to have insufficient sensitivity for the assessment of adult patients who report to the emergency room with non-traumatic abdominal pain [3, 4]; hence a multidetector CT (MDCT) scan is increasingly performed as a first diagnostic step. Consequently, knowledge of the features of LBV on MDCT is important for accurate diagnosis.

\section{Pathophysiology and epidemiology}

Most LBVs are the result of a closed-loop obstruction around a fulcrum point. Genesis of an LBV requires the twist of a mobile loop around a fulcrum point, this

Address correspondence to: Dr Marc Zins, Groupe Hospitalier Paris Saint Joseph, Service d'Imagerie Médicale, 185 Rue Raymond Losserand, 75014 Paris, France. E-mail: mzins@hpsj.fr typically occurs when there is a mobile loop with an elongated mesocolon and a narrowed base, and hence both ends of the loop are close together [5]. Mobile segments that can be involved include the sigmoid, transverse colon and even caecum, which is mobile in $11-25 \%$ of the general population [6]. Figure 1 shows the main types of LBV illustrated as schematic drawings.

The severity and duration of the intestinal and mesenteric obstructive process determines the potential development of complications. During LBV, strangulation of the vascular supply within the twisted mesentery leads to a decreased blood flow and ischemia of the bowel wall. The consequences of ischemia include mesenteric haemorrhage, intramural haematoma, lack of peristalsis and distension, finally leading to infarction with perforation.

Predisposing factors include congenital or acquired anatomical variations, such as a mobile caecum, a long redundant sigmoid with an elongated mesentery, a history of abdominal surgery, late-pregnancy and patient history factors, such as mental retardation, a high-fibre diet, chronic constipation and coincidental disease $[1,5]$.

\section{Multidetector CT technique}

The CT protocol routinely used in our institution for nontraumatic acute abdomen was performed in each case. Rectal administration of contrast enema was performed whenever a colonic obstruction was suspected.

Our protocol for a $64 \mathrm{MDCT}$ scan includes an initial unenhanced low-dose scan of the abdomen and pelvis to exclude pneumoperitoneum (collimation $2.5 \mathrm{~mm}$ ). Stabilised patients then received rectal administration of contrast material, and a second scan with intravenous 


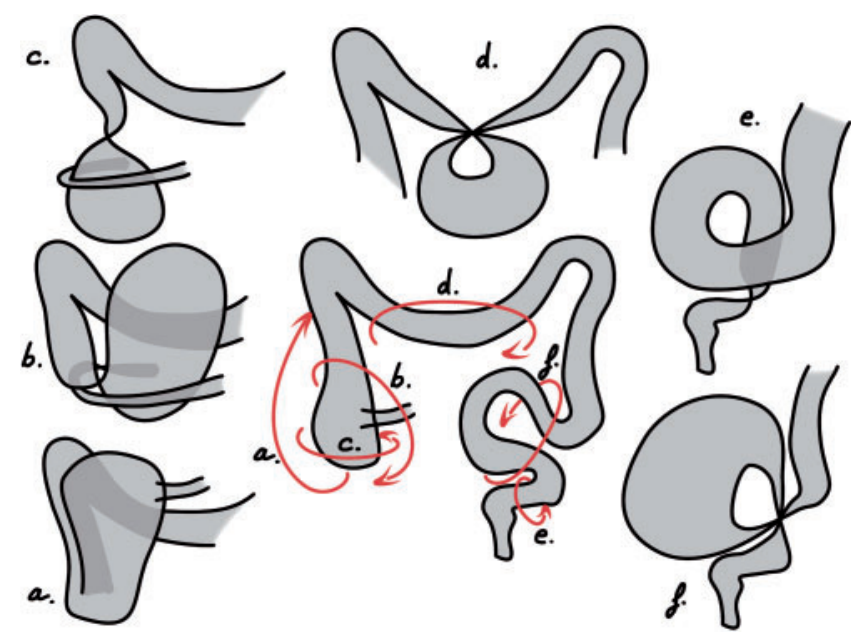

Figure 1. Schematic drawings of the most frequently encountered large bowel volvuli. Each arrow on the diagram of the normal colon represents a possible torsion mechanism, resulting in: a) a bascule type cecal volvulus (CV), b) a loop type CV, c) an axial type CV, d) a transverse colon volvulus, e) an organo-axial sigmoid volvulus and f) a typical sigmoid volvulus.

injection of $120 \mathrm{~mL}$ of contrast material (Iomeron 350, Bracco Imaging SpA, Milan, Italy) delivered at the rate of $2-4 \mathrm{~mL} / \mathrm{s}$ using a power injector was performed at portal phase. Enhanced images were obtained with $0.625 \mathrm{~mm}$ collimation and were reconstructed with a soft-tissue algorithm. The mean total dose-length product (DLP) was $750 \mathrm{mGy} \mathrm{cm}$. The mean duration of a complete MDCT exam was fewer than 10 min. Images were analysed using stack mode and multiplanar reconstructions (MPR) in the coronal and sagittal planes. On-demand three-dimensional reconstructions of selected patients were performed to help the referring physician understand the topography and mechanism involved in the most complex situations.

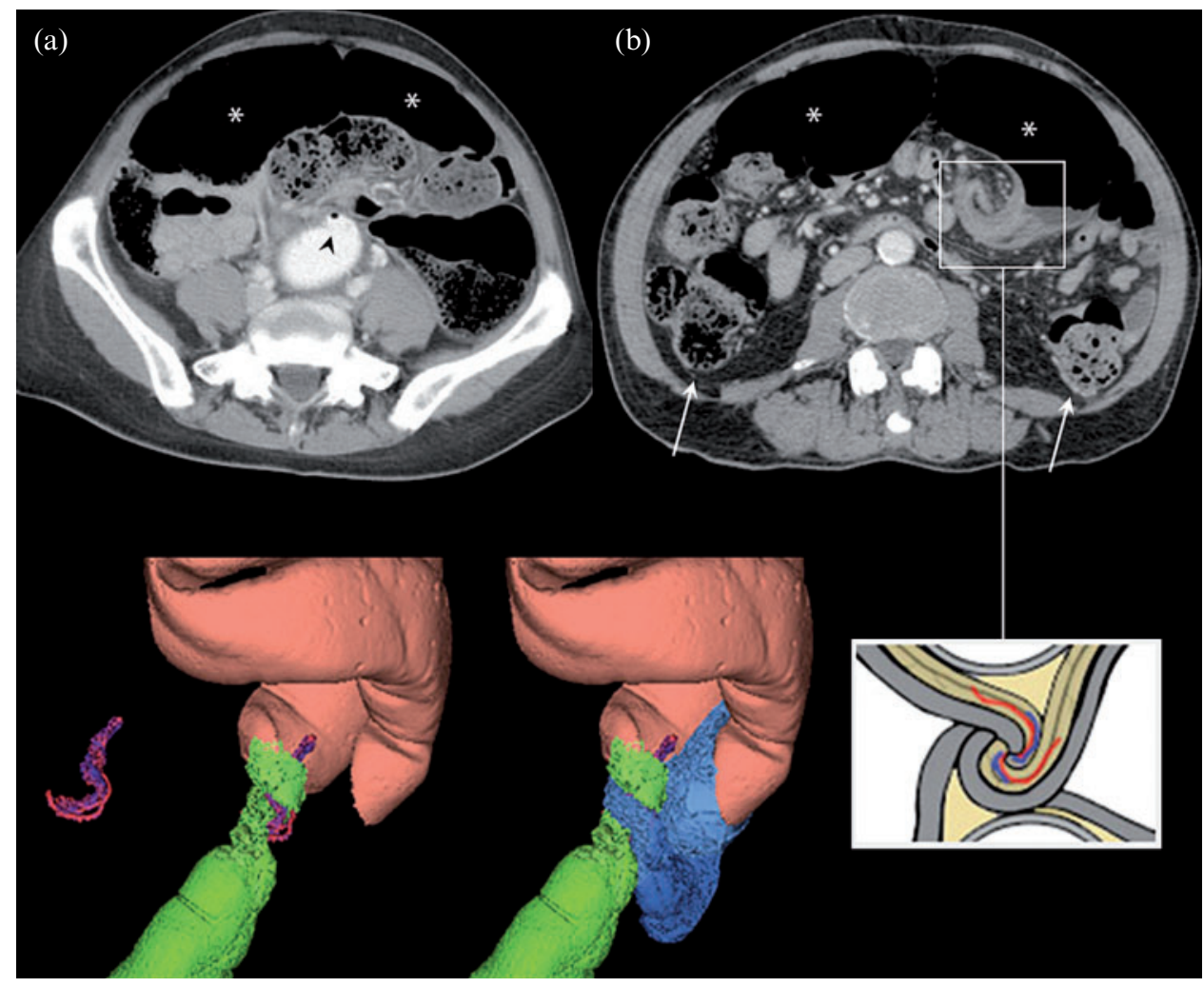

Figure 2. The "whirl sign" and typical appearance of a sigmoid volvulus. (a) Axial CT slice showing a beak sign and no progression of contrast enema beyond the obstruction site (black arrowhead) and the dilated sigmoid loop (*). (b) Axial CT slice centred on the whirl sign of a sigmoid volvulus. Both ends of the dilated loop $\left(^{*}\right)$ can be seen, as can the normal upstream colon (white arrows). The three-dimensional reconstructions illustrate the lateral view of a sigmoid volvulus showing the rectum (green), the twisted loop (pink) and the efferent loop (blue). The drawing illustrates the structures involved in the whirl sign. 


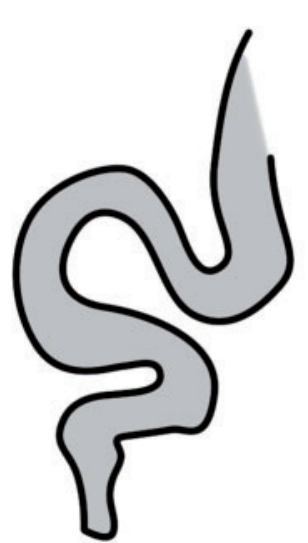

(a)

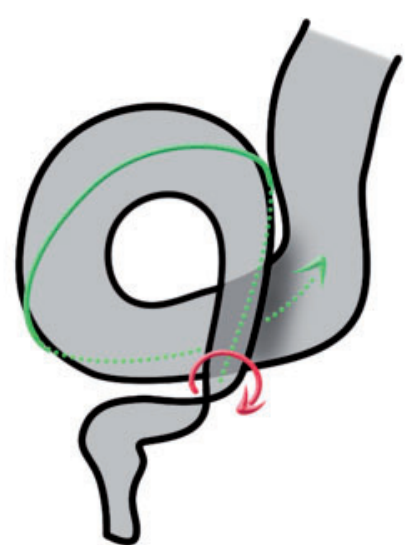

(b)

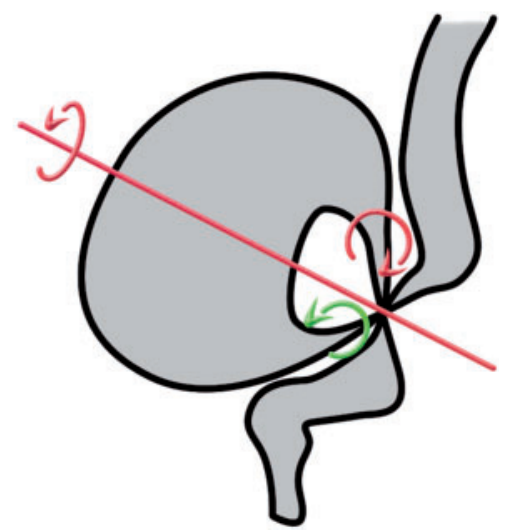

(c)

Figure 3. Sketches illustrating the differences between (a) a normal sigmoid colon and a colon with (b) an organo-axial or (c) a mesenterico-axial volvulus. Arrows represent torsion mechanisms, and the resulting mesenterico-axial axis is shown in (c).

\section{Diagnosis and severity signs}

When demonstrating an LBV the diagnosis criterion is an abrupt transition between a normal and dilated bowel combined with the observation of convergence of both ends of the dilated loop towards the fulcrum point, creating the closed-loop obstruction. An important sign to look for is the "whirl sign", which was first described by Fisher [7]. This is a whirlpool pattern of concentric structures including twisted intestinal loops, vessels and mesenteric fat that is highly suggestive of a torsion mechanism (Figure 2). The whirl sign is visible, however, only if the view plane is orthogonal to the axis of rotation. If the axis of the whirl were in the cranio-caudal axis, for example, the whirl sign would be best visualised in the axial plane. Therefore, we always review our exams using MPR. When administered, the contrast enema does not go beyond the obstruction site and the abrupt interruption appears as a "beak sign".

MDCT helps radiologists to assess the severity of the condition by analyzing the twisted loop wall and the mesentery [8]. Spontaneous increased attenuation of the large bowel wall (which is related to transmural haemorrhagic necrosis), the absence or decreased enhancement of the large bowel wall, pneumatosis intestinalis (i.e. gas in the bowel wall) or thickening of the

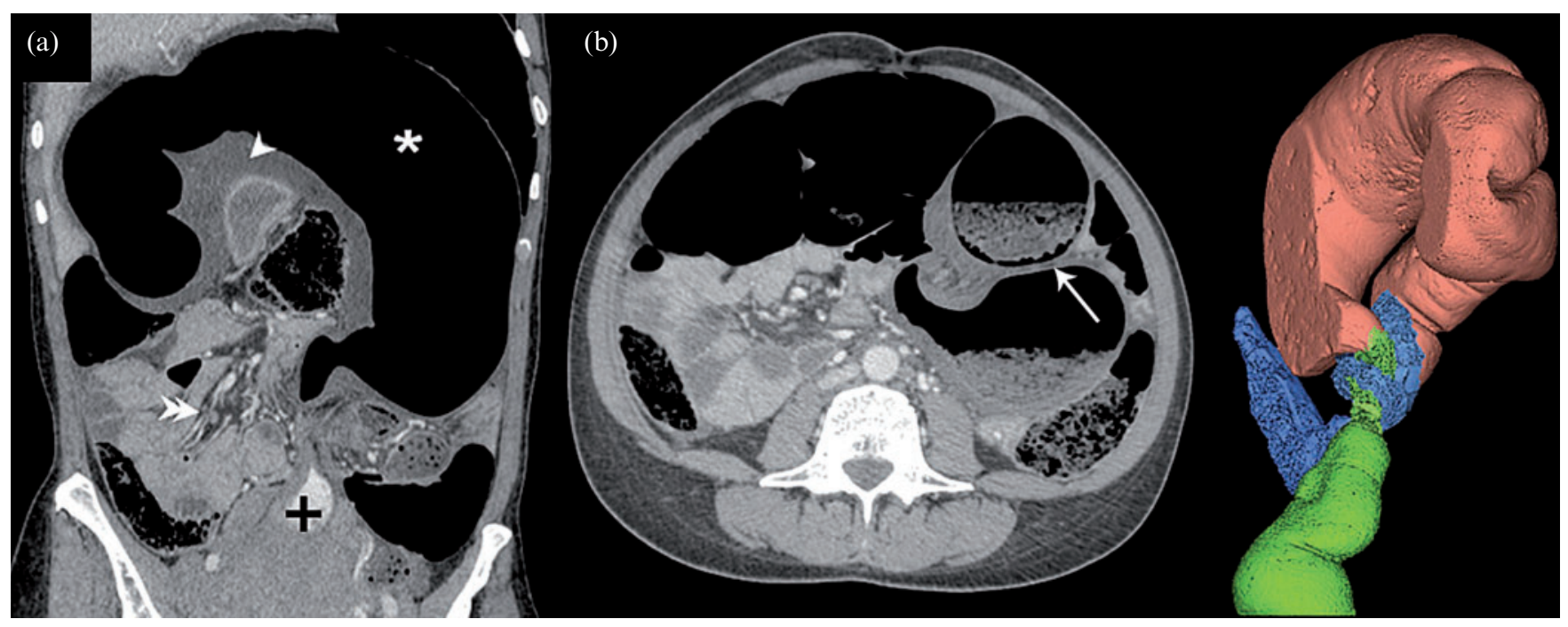

Figure 4. A typical sigmoid volvulus (SV) in a 51-year-old woman. (a) Coronal CT slice showing a typical SV and severity signs: dilated loop $(*)$, rectum filled with contrast enema $(+)$ and fully infiltrated mesosigmoid (arrowhead), which can be compared with normal mesentery (double arrowhead). (b) Axial CT slice at the fulcrum point (cranio-caudal axis). Pneumatosis intestinalis of an afferent limb (white arrow), another severity sign, is seen here. The three-dimensional reconstruction shows a closed sigmoid loop (pink) with both limbs of the twisted loop converging toward the fulcrum point, the rectosigmoid junction (green) and the sigmoid-descending colon junction (blue). 


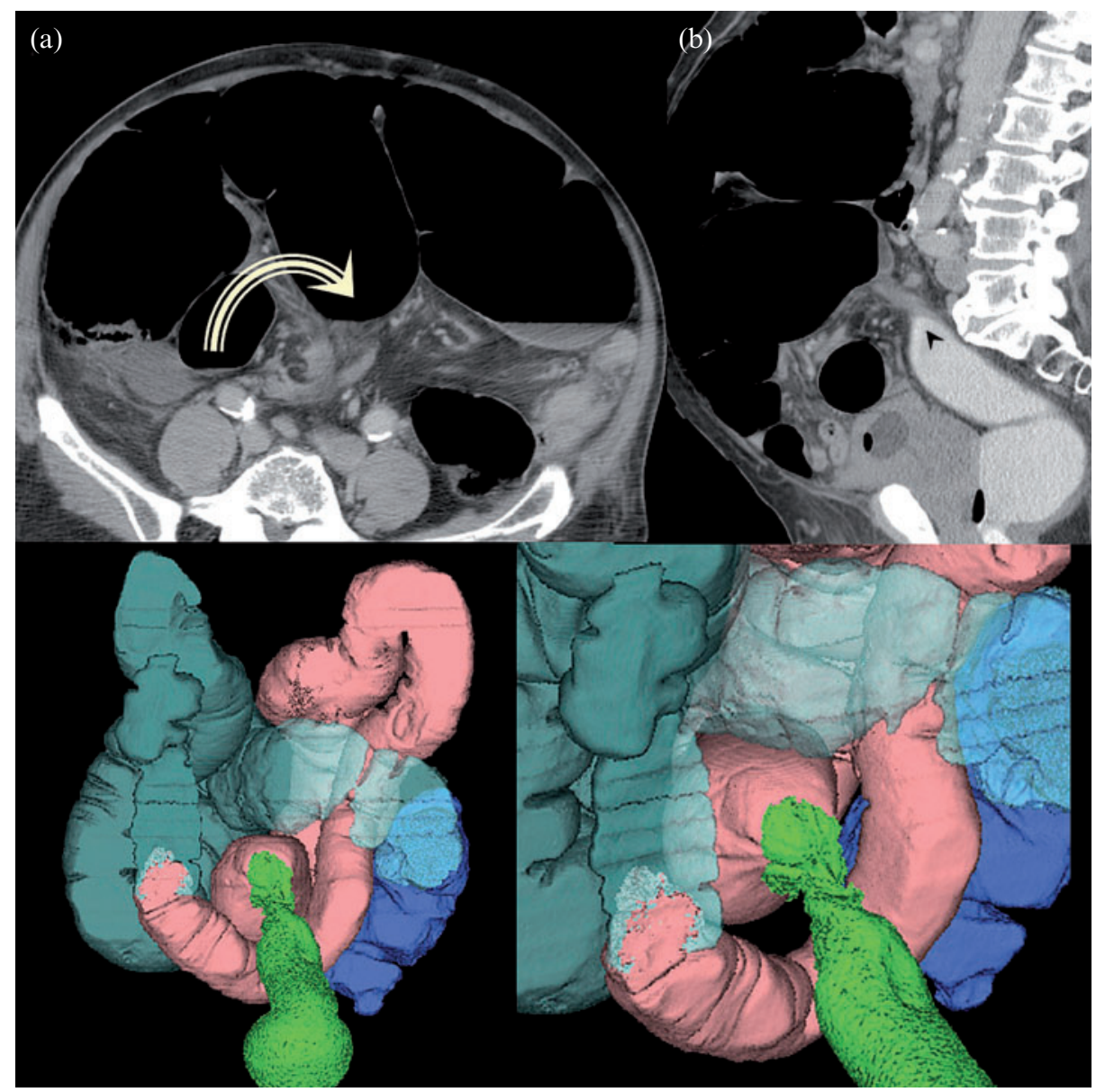

Figure 5. An organo-axial sigmoid volvulus in an 86-year-old man. (a) Axial and (b) sagittal CT slices at the fulcrum point show a low-tight whirl sign (cranio-caudal axis) and a beak sign (black arrowhead). In the three-dimensional reconstructions, the sigmoid (pink) follows the twisted rectosigmoid junction (green). The descending colon junction remains out of the twist and the upstream colon is dilated (light blue and semi-transparent).

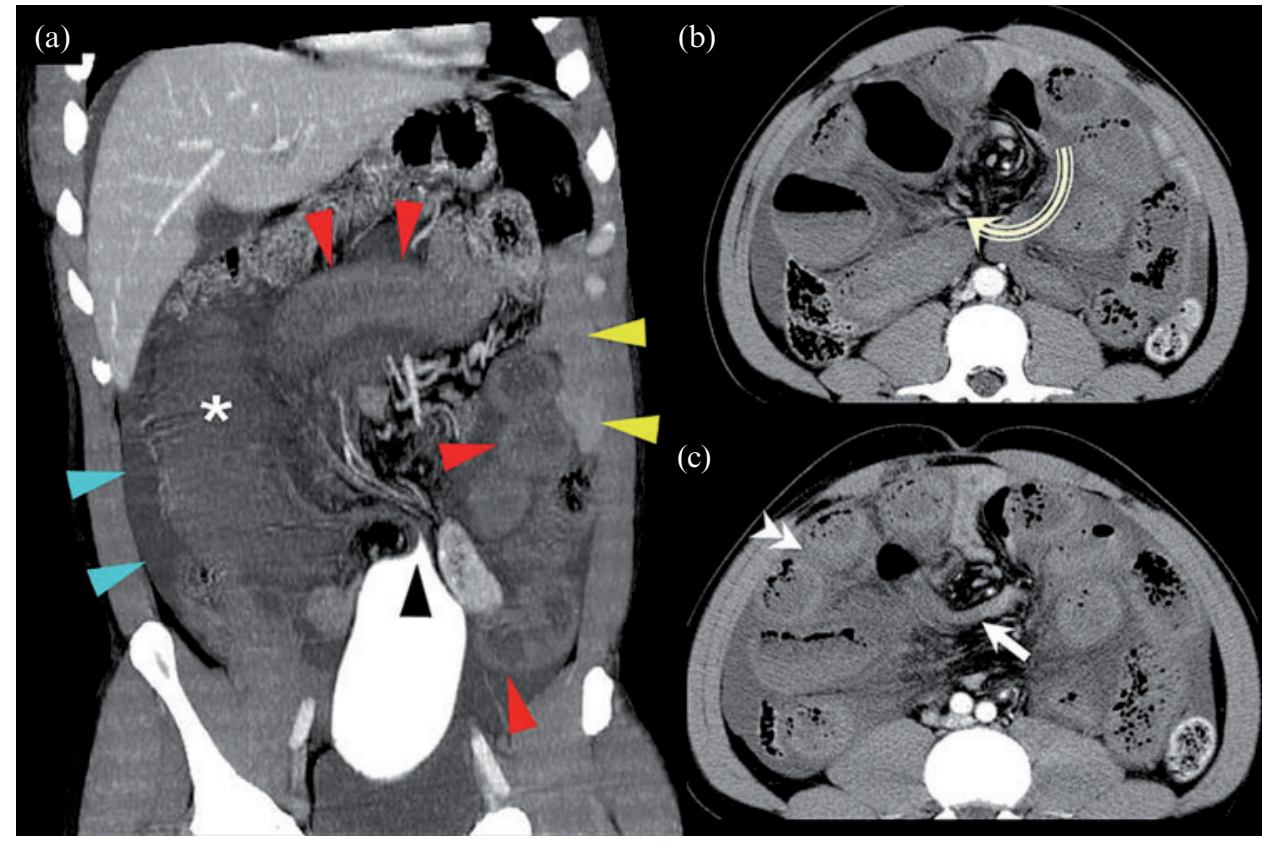

Figure 6. A compound volvulus in a 45-year-old man who presented with signs of shock on admission. (a) Coronal CT slice showing an abrupt stop of contrast enema (black arrowhead), twisted sigmoid (*), twisted ileal loops (red arrowheads), normal small bowel loops in the left flank (yellow arrowheads) and ascites (blue arrowheads). Axial CT slices at the fulcrum point show (b) a large whirl sign (cranio-caudal axis, curved arrow) which includes both limbs of the sigmoid volvulus (arrow) and (c) ileal loops (double arrowhead). Lack of enhancement of the bowel wall after iv injection suggested necrosis. 
large bowel wall each suggest ischemia [6,9] (Figure 4a, $7 \mathrm{a}, \mathrm{b}, 9 \mathrm{a}, \mathrm{b}$ and $10 \mathrm{a})$. MDCT readily demonstrates perforation by showing even small pneumoperitoneum.

CT signs with low specificity for severe forms of LBV are the presence of ascites or haemorrhage, or the absence of vessel enhancement within the twisted loop mesentery $[6,9]$.

Prompt recognition of these findings and quick diagnosis of LBV is mandatory given the high rate of potentially lethal complications [5].

\section{Sigmoid volvulus}

Typical cases of SV (Figures 1f, 3 and 4) present as closed loop obstructions. The loop twists around its mesenteric axis resulting in a "mesenterico axial volvulus".
Both limbs of the twisted loop converge towards a fulcrum point, which appears as a whirl sign when the view plane is orthogonal to the rotation axis of the loop. In most cases, the whirl sign is found in the lower left part of the abdomen with a cranio-caudal axis. The rectum and the upstream colon are usually flat whereas the twisted loop is highly distended and located in the anterior part of the abdomen.

An alternative form of SV is the organo-axial volvulus (Figures 1e, 3 and 5) [10]. In these cases, there is no closed-loop obstruction because only one transition point is seen. Torsion occurs around the long axis of the loop and there is no more than one complete turn in the organo-axial volvulus.

Ileosigmoid knotting, or compound volvulus, is a rare mechanism of SV associated with small bowel volvulus (Figure 6). It is more frequently found in Africa, Asia and

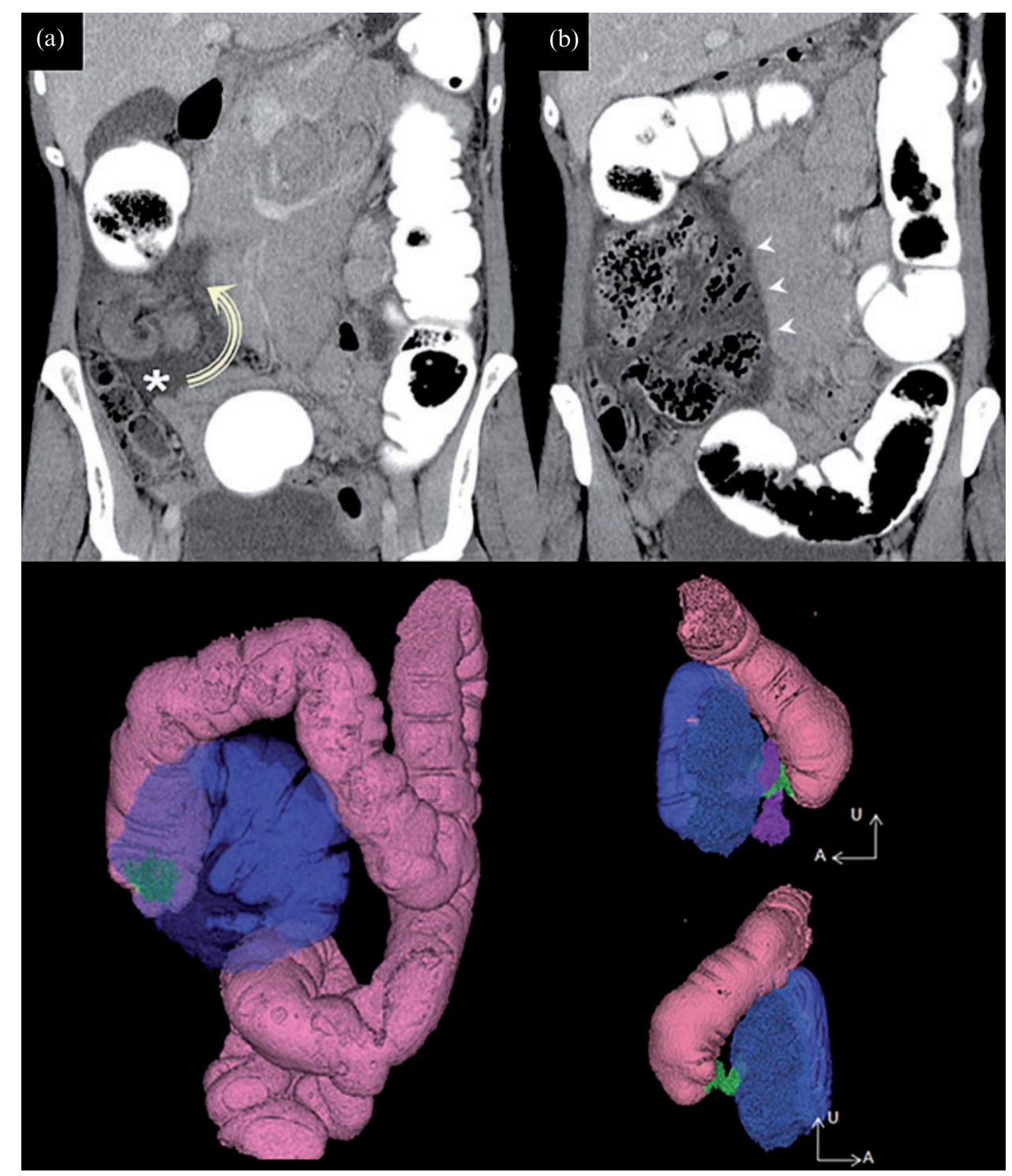

Figure 7. An axial-type volvulus of the caecum in a 56-year-old woman with who presented with signs of shock on admission. (a,b) Coronal CT slices at the fulcrum point demonstrate a tight whirl sign (anteroposterior axis). The caecum remains in the lower part of the abdomen, on the right side. The ileo-caecal region is not involved. There is no free air in the abdominal cavity but severity signs can be seen: there is significant thickening of the caecal wall (white arrowheads) that shows no enhancement and ascites (*) are present. A caecal perforation was present at laparotomy. The three-dimensional (3D) reconstructions show the twist (green) and the colon (pink). The highly distended caecum (blue) has moved up a little, giving the rotation axis an anteroposterior direction. Ileum (purple on top right 3D view) is wrapped around the torsion site but is not included in the whirl sign. 
the Middle East, at an average age of 40 years [11]. The twist involves a sigmoid loop as well as ileal loops. Four limbs converge to the fulcrum point and the whirl sign is larger and located higher in the abdomen [12]. This diagnosis requires emergency surgery because ileal ischemia is prone to quick perforation and leads to a poor outcome.

The initial treatment of SV is generally accepted to be endoscopic decompression by either rigid or flexible sigmoidoscopy [2]. Urgent laparotomy is indicated in the presence of clinical signs of peritonitis or CT signs of bowel ischemia or perforation.

\section{Cecal volvulus}

$\mathrm{CV}$ is the torsion of a mobile caecum around its own mesentery, which often results in a closed-loop obstruction and a distended caecum. The terminal ileum is usually twisted along with the caecum. In most cases of $\mathrm{CV}$, the whirl sign is found in the right part of the abdomen with a lateral or an anteroposterior axis.

Three forms of CV have been described: axial-type CV, loop-type CV and cecal bascule [6, 9]. The axial type
(Figures 1c and 7), in which the caecum rotates along its vertical axis, accounts for $45 \%$ of $C V$ cases. In this type of $\mathrm{CV}$, the distended caecum remains in the lower part of the abdomen, near the right side, which may lead to misdiagnosis on plain radiographs.

The loop type (Figures $1 \mathrm{~b}$ and 8 ) also accounts for $45 \%$ of $\mathrm{CV}$ cases. In these cases, the cecal rotation is associated with an inversion secondary to an anterior displacement. Furthermore, the distended caecum is found in the upper part of the abdomen, near the left side. Finally, the cecal bascule (Figures 1a and 9) is encountered in 10\% of CV cases. No twist occurs in this case, but anterior folding of the caecum, without rotation, is observed and there is no whirl sign in any axis. The distended caecum is found in the upper part of the abdomen, but it remains on the right side.

Management of $\mathrm{CV}$ requires surgical intervention because endoscopic exsufflation is highly unpredictable [13].

\section{Uncommon volvulus}

A volvulus may involve a fixed part of the colon which, in theory, cannot move owing to its retroperitoneal location, fixation by various ligaments or a broad

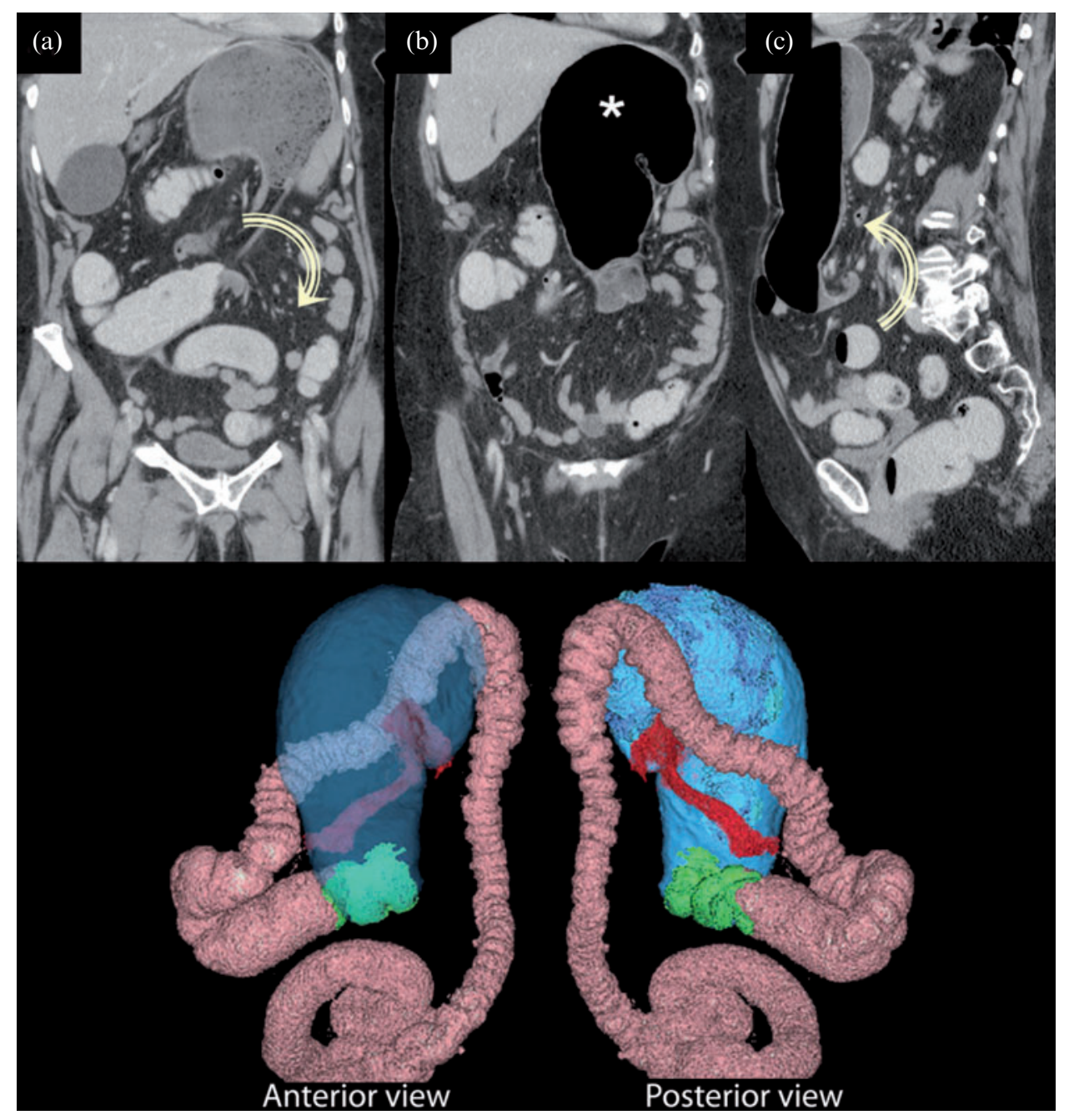

Figure 8. A loop-type volvulus of the caecum in a 74-year-old woman. (a,b) Coronal CT slices from back to front and (c) a sagittal CT slice at the fulcrum point demonstrate a low-tight whirl sign (lateral axis). The caecum (*) is upside down and twisted, and is seen on the left side of the upper part of the abdomen. The three-dimensional reconstructions show the caecum (blue), colon (pink), twist (green) and ileum (red). 


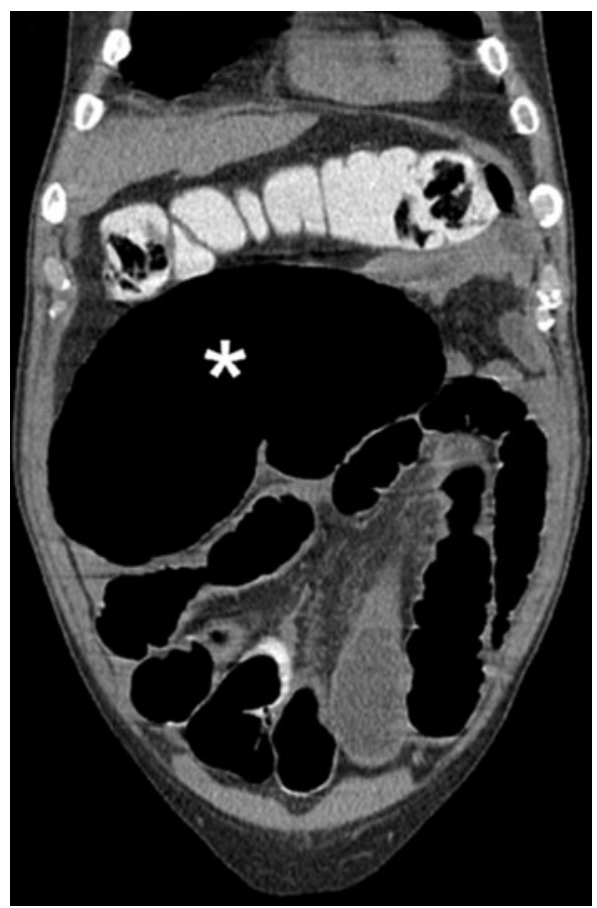

(a)

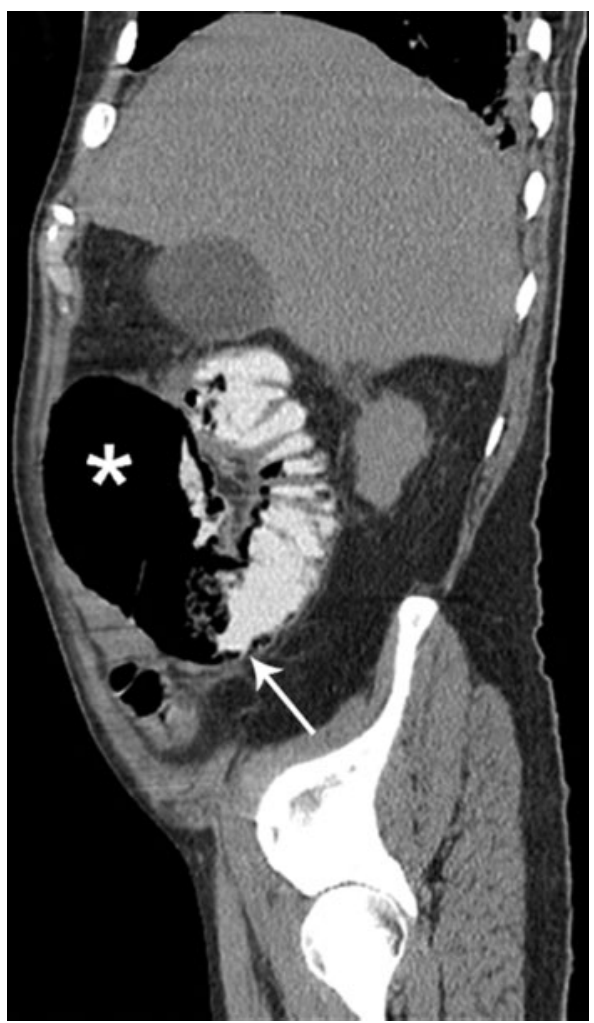

(b)

Figure 9. Caecal bascule in a 60-year-old man. Even though colonic obstruction is not complete, as suggested by contrast enema reaching the caecum, there is evidence of severity with a highly distended caecum $(*)$ visible on (a) the coronal CT slice and (b) the presence of pneumatosis intestinalis of the caecal wall (white arrow). (b) The sagittal CT slice shows anterior folding of the caecum (lateral axis).

base of mesenteric attachment. These segments include the right colon, the transverse colon, the splenic flexure [14] or even the descending colon [15]. Volvulus of the right hemicolon (Figure 10) is exceptional, requiring a persistent mesocolon. To the best of our knowledge, this type of LBV has never been described in the radiological

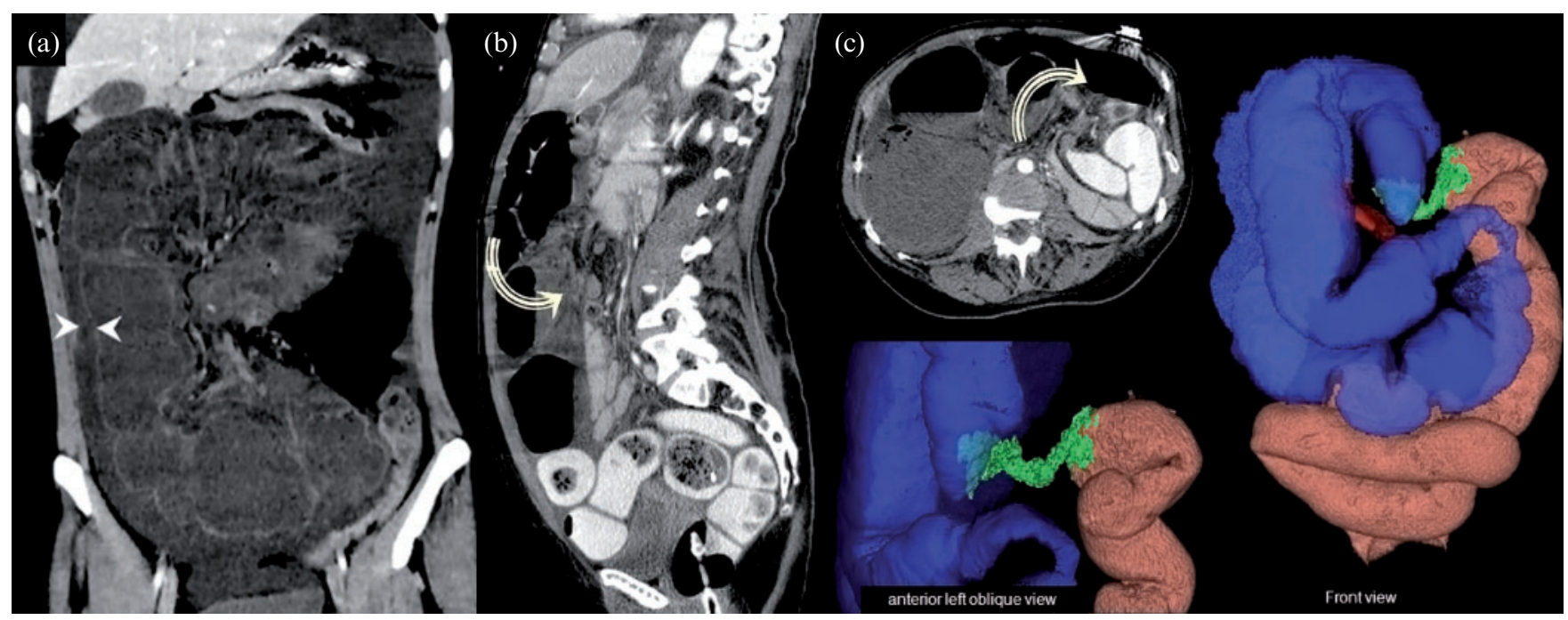

Figure 10. Right hemicolon volvulus in a 59-year-old woman who presented with signs of shock on admission. (a) Coronal, (b) sagittal and (c) axial CT slices at the fulcrum point demonstrate a whirl sign located in the upper and posterior part of the abdomen (lateral axis) and involving the transverse colon. The twisted loop involves the entire right hemicolon (i.e. caecum, ascending colon and half of transverse colon). Severity signs include wall thickening (between arrowheads in (a)) and almost no enhancement of the wall. In the three-dimensional reconstructions, the twist (green) is located in the centre of the transverse colon. The ascending colon and caecum (blue) are in a very unusual position. Thus, the caecum, which can be identified by the terminal ileum (red), is inverted backward. 


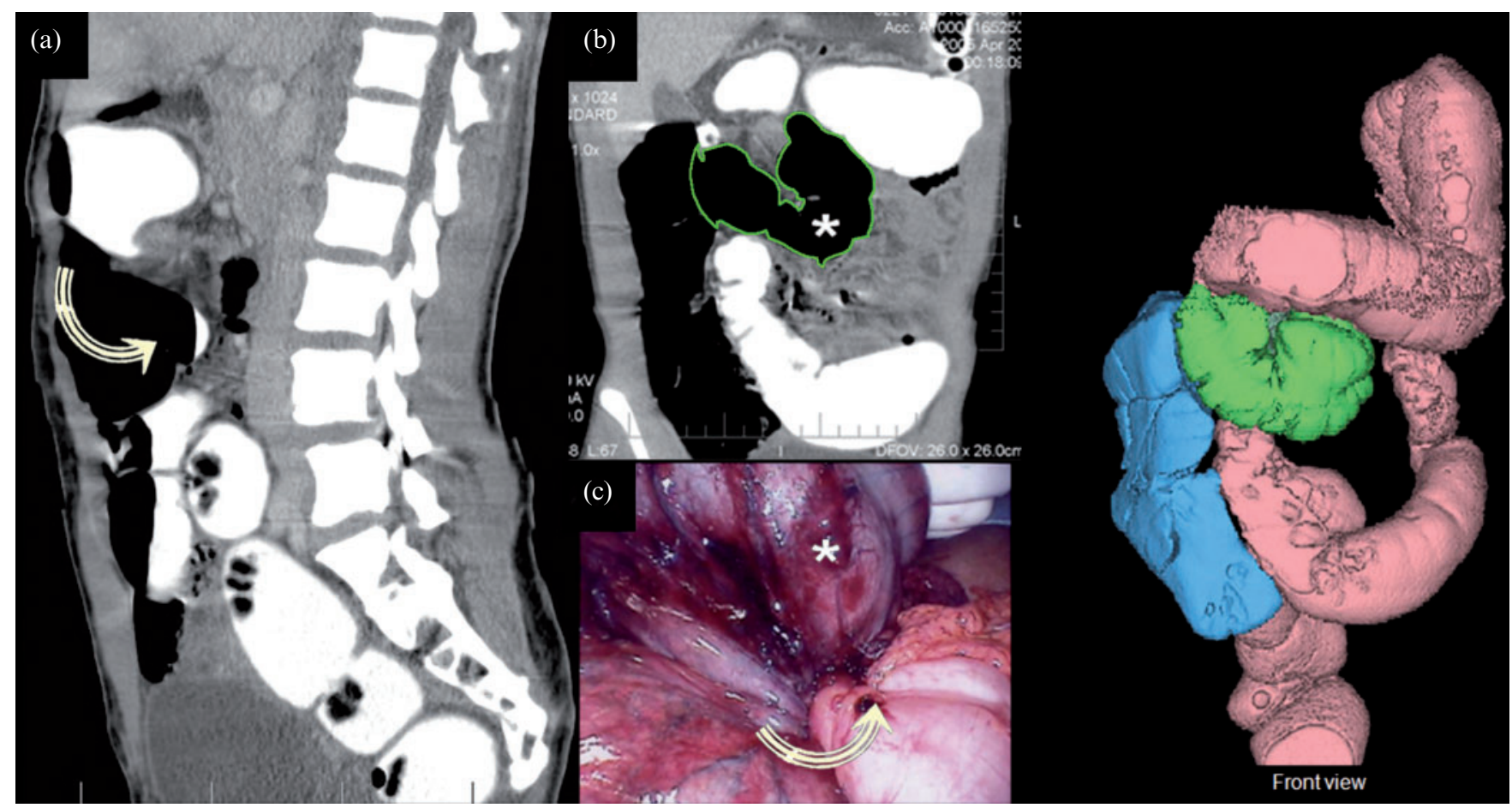

Figure 11. Transverse colon volvulus in a 23-year-old woman. (a) Sagittal and (b) coronal CT slices at the fulcrum point demonstrate a whirl sign that is located in the upper and posterior part of the abdomen (lateral axis). Both limbs of the twisted loop (* with green outline in (b), * in (c)) converge on the transverse colon. The twisted loop involves a segment of the transverse colon. Upstream and downstream colons are seen in the correct position. (c) Peroperative view: free round ligament of the liver creating a strap with no adhesion to the parietal peritoneum. In the three-dimensional reconstructions, the twisted loop (green) is located on the transverse colon. The ascending colon and caecum (blue) are at their usual locations, as are the descending colon and splenic flexure (pink).

literature. Although the transverse colon is a mobile loop, a volvulus in this region of colon (Figures $1 \mathrm{~d}$ and 11) is very unusual because its base is spread widely from splenic to hepatic flexure.

\section{Conclusion}

Volvulus formation is a rare but severe cause of organic obstruction of the large bowel. By establishing a precise diagnosis including topography, mechanism and severity, MDCT allows accurate assessment of large bowel volvuli. Radiologists should be able to recognise their appearance so that the proper diagnosis can be made and catastrophic consequences can be avoided

\section{Bibliography}

1. Jones DJ. ABC of colorectal diseases. Large bowel volvulus. BMJ 1992;305:358-60.

2. Lau KC, Miller BJ, Schache DJ, Cohen JR. A study of largebowel volvulus in urban Australia. Can J Surg 2006;49:203-7.

3. Ahn SH, Mayo-Smith WW, Murphy BL, Reinert SE, Cronan JJ. Acute nontraumatic abdominal pain in adult patients: abdominal radiography compared with CT evaluation. Radiology 2002;225:159-64.

4. Kellow ZS, MacInnes M, Kurzencwyg D, Rawal S, Jaffer R, Kovacina $\mathrm{B}$, et al. The role of abdominal radiography in the evaluation of the nontrauma emergency patient. Radiology 2008;248:887-93

5. Madiba TE, Thomson SR. The management of cecal volvulus. Dis Colon Rectum 2002;45:264-7.

6. Moore CJ, Corl FM, Fishman EK. CT of cecal volvulus: unraveling the image. AJR Am J Roentgenol 2001;177:95-8.

7. Fisher JK. Computed tomographic diagnosis of volvulus in intestinal malrotation. Radiology 1981;140:145-6.

8. Catalano O. Computed tomographic appearance of sigmoid volvulus. Abdom Imaging 1996;21:314-7.

9. Delabrousse E, Sarlieve P, Sailley N, Aubry S, Kastler BA. Cecal volvulus: CT findings and correlation with pathophysiology. Emerg Radiol 2007;14:411-5.

10. Janzen DL, Heap SW. Organo-axial volvulus of the sigmoid colon. Australas Radiol 1992;36:332-3.

11. Kotisso B, Bekele A. Ilio-sigmoid knotting in Addis Ababa: a three-year comprehensive retrospective analysis. Ethiop Med J 2006;44:377-83.

12. Lee SH, Park YH, Won YS. The ileosigmoid knot: CT findings. AJR Am J Roentgenol 2000;174:685-7.

13. Habre J, Sautot-Vial N, Marcotte C, Benchimol D. Caecal volvulus. Am J Surg 2008;196:e48-9.

14. Mittal R, Samarasam I, Chandran S, Mathew G. Primary splenic flexure volvulus. Singapore Med J 2007;48: e87-9.

15. Chen A, Yang FS, Shih SL, Sheu CY. Case report. CT diagnosis of volvulus of the descending colon with persistent mesocolon. AJR Am J Roentgenol 2003;180: 1003-6. 